\title{
SELF-ORGANIZATION AND EMERGENCE IN GLOBAL HEALTH. INSIGHTS FROM PRACTICE, BLIND SPOTS AND POSSIBLE CONTRIBUTIONS FROM COMPLEXITY SCIENCE
}

\author{
E.G. SARRIOT ${ }^{1}$, E.R. SACKS ${ }^{1} \&$ A. LARSON ${ }^{2}$ \\ ${ }^{1}$ ICF International Center for Design and Research in Sustainable Health and Human Development (CEDARS), USA \\ ${ }^{2}$ National Centre for Epidemiology and Population Health, The Australian National University, Canberra, Australia
}

\begin{abstract}
Global health as a discipline is at a moment of tremendous achievements and general optimism, but faces enduring questions, notably about the sustainability of its endeavours. The field of global health defines itself through enumerated goals, targets, and indicators to track progress of complex changes. With this comes a broad panoply of objective-driven strategies resting on assumed control over chains of cause and effect. We present areas where emergence and self-organization play an important role in determining the sustainability of positive outcomes at scale and behaviours of both the population and its service providers. The role of self-organization and emergence seem under-appreciated by the field and we consider three reasons for a possible "blind spot" of global health decision makers and influencers: (1) the culture of command and control inherent to central planning, (2) the structural limitations of large complex institutional edifices in processing information (variety), and (3) epistemological/cognitive constraints. More pro-active allowance for the conditions of positive self-organization and emergence may be required to avoid unintended and negative long-term consequences of global strategies, in spite of best intentions. Observational, analytical and computational multi-disciplinary studies are needed to bring clarity to these questions, and to inform a parallel process of dialogue, provocation, and learning to evolve the institutional culture of global health at both national and international levels.

Keywords: agent-based modelling, complex adaptive systems, computational sciences, emergence, global health, self-organization, viable system model.
\end{abstract}

\section{INTRODUCTION}

Global health has emerged in the last 20-30 years into its own field. Its goals are to solve the largest mortality and morbidity problems globally, and address health disparities [1]. It builds on decades of international health and international development, and brings together a wide range of actors: country institutions, government-to-government programs, United Nations, non-governmental agencies (NGOs), public-private partnerships, etc. Global health's culture emphasizes performance, effectiveness, accountability, country ownership, and evidence-based interventions [2,3]. This has come with notable progress on reducing child, neonatal, and maternal mortality, and towards disease control and expansion of services. This evolution is part of the Millennium Development Goals (MDGs), which were partially achieved by their 2015 target, and now, the Sustainable Development Goals (SDGs) [4]. Global health has achieved great success overall and is going through a time of relative optimism about what can be achieved in the next generation [3, 4].

Some caution is however warranted for at least two reasons: First, mortality gains are due to far more than effectiveness in pursuit of defined goals [5, 6]. Progress towards the MDGs were achieved during a period of increase in gross domestic product (GDP) in developing countries, and an important part of the gains in child mortality are associated with GDP gains and social determinants of health 
rather than specific health interventions supported by global health strategies [5, 7]. Secondly, most progress has been made in a context of increased development assistance [7, 8], and this raises questions about the sustainability at scale of systems, of quality, and of health outcomes $[9,10]$.

These questions have stimulated a growing body of literature on complexity and systems thinking in global health $[11,12]$, which this paper adds to, to make three points: emergence and self-organization play an important role in achieving and sustaining global health results (section 2); global health is constrained in its capacity to discern these phenomena (section 3); finally, section 4 discusses a way forward, in terms complex systems research, and in terms of our cultural evolution.

\section{EMERGENCE AND SELF-ORGANIZATION IN GLOBAL HEALTH}

We refer readers to the literature for a definition of the related but different concepts of self-organization and emergence $[13,14]$. We use the limited literature on self-organization and emergence in global health to make the case that these phenomena play a greater role in global health than usually acknowledged, and identify examples at three levels:

\subsection{Self-organization in community and women's groups}

Two models for improving community health - Participatory Women's Groups (PWGs) and Care Groups (CGs) - have drawn attention recently with large scale international implementation and a substantial level of evidence $[15,16]$. The models bring women together in social structures to address specific health issues. The similarities and differences in the models are beyond our scope. Promoters of these approaches have defined the models to allow replicability and building an evidence basis. But informal discussions with authors, if not in the publications themselves, suggest at least the potential for emergence and self-organization in the participatory and social interactions' elements of these models. Successful women's groups evolve, adjust behaviours, and take on new tasks or objectives over time and of their own volition. Longitudinal studies are not available, but these unanticipated self-organizing processes seem to increase with time - more tasks, new roles, complex group dynamics. Whether this supports initial goals or not, self-organization seems to be a factor in these group approaches.

\subsection{Self-organization within the health workforce and between health workers and clients}

We identified different examples of self-organization in the literature: health providers with formal day-time functions in the health system and informal clinical practice at night [17]; community health worker groups developing new functions (savings activities) [18]; and district managers strategies to deal with the unreliability of central funds [19].

Less documented is the use and misuse of per diems for health workers. In the context of development, external actors can sponsor activities by providing in kind or financial (per diem) compensation. This can both stimulate and disrupt planned activities [20]. But self-organization can also be used to correct such unintended effects. In two cases (undocumented), management teams systematically redistributed part of the per diems to incentivize staff who failed to benefit from these payments. Rather than establishing patronage, local self-organizing redistributed incentives more equitably.

Finally, Lanham [21] describes initiatives, which "took advantage of self-organization" between health workers and clients to strengthen scale-up strategies. An intervention using cellular messaging to increase compliance to treatment saw unplanned uptake of phone communication between patients and nurses, intervention group patients relaying messages to control group friends; and spontaneous formation of a support network of patients. 


\subsection{Scaling and sustaining global health achievements}

Decentralized management and autonomy can be hard to distinguish from actual self-organizing in the literature $[22,23]$. But we find examples of self-organization and emergence in publications on sustaining health outcomes: Sarriot [24] surveyed primary health care projects' implementers, and reported maintenance of some activities coming through unanticipated local processes. Program managers referred to supporting "a local process - particularly at the community level - without being able to predict or control it... it was completely unintentional in terms of where we would go next with it . . . It wasn't exactly a mistake, but it wasn't necessarily an intended strategy". An assessment five-year post-project [25] describes elements of self-organization among stakeholders of an urban health project in Bangladesh. While on the surface the architecture for urban community health was as-expected, different groups' self-interest led to a working equilibrium between all parties [26].

Similar language is used for scale-up processes [27]. ExpandNet, a World Health Organization collaborative, is dedicated to advancing scale-up of evidence-based health strategies, and recognizes the existence of spontaneous scaling up, as "diffusion of the innovation without deliberate guidance" [28]. Plausible examples of self-organization can be found where actions by national, local governments and community leaders changed the course of a pre-determined scale-up strategy [29]. Another series of case studies on scaling essential life-saving interventions describes self-organizing behaviours [30]: communities spontaneously providing homes and food to community health workers; and a program to expand access to post-partum family planning methods in India finding that health facilities combined planned and self-organized peer training of nurses to achieve scale.

These phenomena echo what is described in other fields of organizational and social sciences. The issue is now which factors hinder their perception by the global health community. We suggest three reasons.

\section{CULTURAL, STRUCTURAL, AND EPISTEMOLOGICAL BIASES AS BLIND SPOTS}

3.1 The international development culture - inherent and natural bias for control and predictability in decision-making

Policy makers require predictability from implementers, and are held accountable for return on investment by their own boards and governments. Objectives and targets translate into command and control approaches. Self-organization, by nature, runs counter to predictability. Additionally, emergence in complex adaptive systems depends on ill-defined timelines, which are poorly reconciled with deadlines.

One of our authors participated in an aid organization workshop, convening experts to address how complexity affected their work. The day saw substantial discussions about local systems' behaviours, adaptive processes, emergence, and how the organization might need to adapt its approaches. Coming to conclude the deliberations of the day, a high level leader of the institution specifically reminded participants that while adaptation to local contexts might be important, there could not and should not be ten different ways of achieving success on global targets. At another meeting, the leader of a network of implementing organizations was describing how trust and shared space within the network had allowed different partnership configurations to emerge and to respond to different challenges (for example Ebola). While sympathetic to the accomplishments, a donor agency manager present concluded the discussion by stating "unfortunately, we cannot fund it 
depends"". These examples echo the words of Lanham [21], "the designs of [scale-up and spread] initiatives tend to focus on reproducing interventions with total fidelity, overlooking the unique attributes of local contexts [...] failed interventions could be the result of overlooking the limits of strict adherence to an intervention design."

\subsection{Structure - the challenge of variety engineering}

Institutional hierarchies require that information be processed and reduced as it moves upstream. Global health has recognized, central, and influential nodes in its network. Harmonization of indicators allows the relative few in the relative centre to process signals coming from nearly 200 countries, and thousands of districts. The diversity of experiences must be reduced level by level in order to be managed. A balance needs to be found between the quantity of signals and information (or "variety" [31]) flowing up from one level of management to the next. Self-organization and emergent phenomena generate increased variety (complexity and signals), which naturally challenge the management capacity of each level of a control hierarchy. It becomes easier for the institutional system to retrospectively describe self-organization either as "happy accidents" (exceptional leadership, serendipity) or unintended consequences (poor coordination, corruption), essentially as surprises outside of implementation research considerations, leaving the signals received by the centre primarily about anticipated processes.

The systematic study of the success factors for countries on the "fast-track" to achieving the MDGs [5] combined data from up to 144 countries. It found that "no single configuration of factors proved necessary or sufficient" to predict success. Factors identified fit broad categories: multi-sector gains including very prominently woman's education, woman's participation in both workforce and political structures, mobilization of partners across society around evidence-based strategies, using evidence for accountability, rapid adaptation to change for course correction, and the use of guiding principles. This does not demonstrate self-organization, but it certainly suggests conditions leading to decentralized intelligence, and responsive adaptation.

\subsection{Knowledge - increased complexity and epistemological challenges}

Contextual complexity challenges knowledge and knowledge management in development efforts [21, 32-34] and global health itself have become more complex (through scale, number of interventions, diversity and interconnections of actors,) [12, 35-37]. This challenges how to make sense of strategies and their evaluation. Jackson [38] presents decision-making and sense-making along two axes:

1. Participants can be characterized as unitary (sharing values, beliefs and interests), pluralist (different values and interests, with consensus on discrete objectives), or coercive (interests differ and power is a driving force); and

2. Systems, which can be characterized as simple (relatively stable, limited number of structured interactions, unaffected by external influences) or complex (large number of subsystems, adaptation to each other, and a shifting environment).

Global health remains often conceptualized as unitary and simple: health is a universal value; evidence-based technologies need to be scaled up with reasonable but limited adaptation to context; priorities are established in national and global fora with correspondingly, clear and objective metrics. Global health may however be more often realized at different levels on the two axes: progress 
takes place in a pluralist environment, where countries have different priorities, based not only on epidemiology, but also the will of the people, the competing interests of a national economy, decentralized powers, and negotiated arrangements between sectors. The environment can also be unwittingly, coercive. Governments have to align to global decision makers who overwhelmingly control finances (i.e. challenging decisions about new vaccines versus routine immunization as just one example). Similar processes affect national to subnational negotiations. Interestingly, in the development field, the complexity matrix describes how specific problems fall on a continuum from simple to complex, and has been adapted to community health [10,37]. As complexity accrues and the world becomes pluralist or coercive, the definition of the goal to be pursued and the metrics of progress start relying on more constructivist and participatory methods for sense-making [10, 21] to answer questions such as, can we sustain a level of collaboration required to maintain equitable health outcomes in communities?

Nurtured by medicine and epidemiology, global health has learned to rely on controlling statistically for contextual factors influencing the effectiveness of new technologies. But trying to simply control for context in evidence generation may inappropriately filter out important phenomena, thus contributing to "blind spots". Anderson [14] states: "The constructionist hypothesis breaks down when confronted with the twin difficulties of scale and complexity. At each level of complexity entirely new properties appear. Psychology is not applied biology, nor is biology applied chemistry. We can now see that the whole becomes not merely more, but very different from the sum of its parts." We should continue the trail and argue that complex social and institutional interventions are not applied medicine and epidemiology. Behaving as if that they were may cause substantial distortions in our understanding.

\section{DISCUSSION: WHAT PATH AHEAD}

The global health community has been extremely dynamic in its speed of change over the last decades but if our argument holds, cultural, structural and epistemological biases risk depriving a professional community from lessons, which may have critical impact on the sustainability of its efforts. How can we move forward?

\subsection{Possible contributions from complex systems science}

There is a growing body of literature on systems thinking and complexity in health [11, 12], sustainable development, and development assistance $[8,23]$. This trend is welcome and should help us move forward.

Exploratory and diagnostic efforts are needed. In our examples, we have had repeated back and forth asking, "is this example really self-organization, or is it something else?" When is local organization self-organization? Our discussions navigated along a continuum, with-on the one end-system behaviours clearly fitting a command and control strategy, others needing marginal adaptation of central strategies to local conditions with global designs still dominating the solution landscape, and finally central evidence-based solutions requiring far more than adaptation. At this point, strategies may need to create space for self-organization. Studies should explicitly examine the how, when, why and impact of self-organization. Mechanisms for decentralized management, autonomous team and peer-learning, and use of performance-based incentives would benefit from "inside the black box" studies. There are a number of other issues, which could test when our hypothesis holds or is disproved [39-44]. Complex systems specialists should play an important role in translating study data in terms of underlying complex adaptive systems behaviours [21, 45-47]. 
Agent-based modelling (ABM) provides another direction to study the behaviours of health workers and social groups in different settings. Agent_Zero [47], for example, provides a template primed for adaptation to our questions. The model assigns agents with deliberative/cognitive and affective/emotional components of a disposition to act. Different environmental stimuli can affect their affective component, and social ties allow disposition contagion. We cannot do justice to the model here, but adaptations of the model could examine the disposition to perform of community group members, community health workers or facility-based care providers. Possible research questions could include how the cumulative aggregate performance of agents evolves under conditions, determined by environment signals and emotional response to financial and non-financial incentives, social signalling (perceived fairness), and cognitive support mechanisms (peer support, supervision, etc.). The model could also be designed to test the long-term dynamics (sustainability) of social/health groups under different types of support. ABM would allow to explore whether patterns of system level performance emerge (regularities) based on environmental, emotional and social signalling, and possibly counter-intuitively to the intentions of the environmental signals (payment and control schemes). Interdisciplinary research could combine the knowledge of computational, behavioural, health care, and social systems scientists with implementers' experiences.

Finally, complex systems science may also offer approaches for testing, on large data sets or in simulations, probabilistic models of success, based on learning from traditional methods. For example, the Sustainability Framework [10] suggests a set of conditions, which if present at a certain level, will increase the odds of emergence of sustainability. These include: policies, specific capabilities, social capital, community capacity, macro-economic conditions, conflict, political stability, feasibility and acceptability of interventions. These factors naturally affect path dependence for how levels of sustainability can be achieved. For example, a certain set of capabilities in a central governmental health institution may be required only under a specific central-decentralized functional distribution of roles. An essential health district capacity in one context may become obsolete in another where civil society organizations are implicitly and explicitly granted public service responsibilities. Simulation models informed by solid case studies could test different configurations of conditions required to achieve success (this may fit something similar to a percolation model, where success is probabilistic and subject to a tipping point).

The implications for global health planners and researchers are important [46]. Under a linear model, the objectives are to maximize progress on the proximal determinants of success, as defined by the largest and most significant coefficients. If sustainability (or scale) follows a probabilistic model instead (for example, a percolation model) then the goal is to advance enough positive conditions while respecting principles for positive self-organization, without trying to individually predict what will be achieved on each proximal determinant.

\subsection{Institutional and cultural evolution}

New studies can provide information and signals, but are probably not enough to bring change in the complex adaptive system of development institutions [8]. Power, influence, trust, and network dynamics within development institutions are beyond this paper, but will influence the speed of learning [48]. Processes of dialogue, provocation, reaction and consensus, along with innovation and research will be needed to translate learning into new behaviours. Principles, such as Axelrod and Cohen's "harnessing complexity" [47] may help not only global health and development practice, but also for evolving the model and behaviours of global health institutions themselves. 
The evolution of new forms of governance and the Paris Declaration for Aid Effectiveness are positive examples for how global health is able to evolve. Our argument is however not simply for more national leadership in development, even if this is a natural starting point. Our call is for recognition that non-linear phenomena based on decentralized agency affect our successes and failures. Our call for exploration and learning goes to both national and international leaders and the research community.

\section{CONCLUSION}

This paper has tackled and tried to relate together a complicated set of questions. In so doing, it has unavoidably been cursory in its treatment of important issues. The argument rests on three parts: (1) that self-organizing and emergent phenomena affect global health achievements, particularly their sustainability at scale; (2) that the leaders of the field of global health face inherent cultural, structural and cognitive challenges in perceiving signals about the potential importance of these phenomena and (3) that multi-disciplinary research directions, notably through contributions of complex systems science, will be necessary, but not necessarily sufficient, for the global health sector to better address these issues.

Both individual and institutional agency weighs heavily in the final outcomes that central planners are investing in. Our contention is not that self-organization is some sort of panacea. Self-organization eventually takes place in human complex adaptive systems for better or for worse. Where self-organization and emergence have a positive role to play, ignorance of their potential opens the field of global health to both opportunity costs and unintended effects. "Where, when, and how does self-organization need to play out?" are the questions we need to ask. Learning how to do this could have a fundamental importance for resolving intractable problems, establishing more resilient social and health systems, and sustaining current achievements in global health. Partnership efforts between complex systems science and global health researchers could help national and global decision makers learn to create space for probabilistic (stochastic) processes within their deterministic plans, lowering the focus on individual program success but creating conditions for greater and more lasting overall impact.

\section{ACKNOWLEDGEMENTS}

We wish to thank reviewers, Sharon Arscott-Mills, Angie Brasington, David Bishai, Ligia Paina, and Inger Scheel. Thanks to Leo Ryan and Ani Hyslop for their support.

\section{REFERENCES}

[1] Hafner, T. \& Shiffman, J., The emergence of global attention to health systems strengthening. Health Policy and Planning, 28, pp. 41-50, 2013. http://dx.doi.org/10.1093/heapol/czs023

[2] OECD, Aid Effectiveness 2005-10: progress in implementing the Paris declaration, OECD Publishing, pp. 1-200, 2011.

[3] USAID, Acting on the call: ending preventable child and maternal deaths report. In United States Agency for International Development (USAID), pp. 1-85, 2015.

[4] United Nations General Assembly, Transforming our world: the 2030 Agenda for sustainable development. In United Nations, New York, NY, pp. 1-35, 2015.

[5] Kuruvilla, S., Schweitzer, J., BIshai, D., Chowdhury, S., Caramani, D., Frost, L., Cortez, R., Daelmans, B., De Francisco, A., Adam, T., Cohen, R., Alfonso, Y.N., Franz-Vasdeki, Y., Saadat, S., Pratt, B.A., Eugster, B., Bandali, S., Venkatachalam, P., Hinton, R., Murray, J., Arscott-Mills, S., Axelson, H., Maliqi, B., Sarker, I., Lakshminarayanan, R., Jacobs, T., Jacks, 
S., Mason, E., Ghaffar, A., Mays, N., Presern, C. \& Bustreo, F., Success factors for reducing maternal and child mortality, pp. 533-544, 2014.

[6] Fukuda-Parr, S., Ely Yamin, A. \& Greenstein, J., Synthesis paper - the power of numbers: a critical review of mdg targets for human development and human rights, 2013.

[7] French, D., Did the millennium development goals change trends in child mortality? 2015.

[8] Ramalingam, B., Aid on the Edge of Chaos. Rethinking International Cooperation in a Complex World, Oxford University Press, 2013.

[9] Chambers, DA., Glasgow, RE. \& Stange, KC., The dynamic sustainability framework: addressing the paradox of sustainment amid ongoing change, 2013.

[10] Sarriot, EG, Kouletio, M, Jahan, S, Rasul, I. \& Musha, A., Advancing the application of systems thinking in health: sustainability evaluation as learning and sense-making in a complex urban health system in Northern Bangladesh. Health Research Policy and Systems, 12(45), 2014.

http://dx.doi.org/10.1186/1478-4505-12-45

[11] Peters David, H., The application of systems thinking in health: why use systems thinking? Commentary, 2014.

http://dx.doi.org/10.1186/1478-4505-12-51

[12] Ramalingam, B., Aid on the Edge of Chaos. Rethinking International Cooperation in a Complex World, Oxford University Press, 2013.

[13] Miller, JH. \& Page, SE., Complex Adaptive Systems: An Introduction to Computational Models of Social Life, Princeton University Press, 2007.

[14] Anderson, P.W., More is different. Science, 177, pp. 393-396, 1972.

http://dx.doi.org/10.1126/science.177.4047.393

[15] Paul, VK., Participatory women's groups: time for integration into programmes. The Lancet Global Health, 4, pp. e74-e75, 2016.

http://dx.doi.org/10.1016/S2214-109X(16)00010-3

[16] Perry, H., Morrow, M., Borger, S., Weiss, J., DeCoster, M., Davis, T. \& Ernst, P., Care groups i: an innovative community-based strategy for improving maternal, neonatal, and child health in resource-constrained settings, pp. 358-369, 2015.

[17] Paina, L., Bennett, S., Ssangooba, F. \& Peters, DH., Advancing the application of systems thinking in health: exploring dual practice and its management in Kampala, Uganda. Health Research Policy and Systems, 12(41), 2014. http://dx.doi.org/10.1186/1478-4505-12-41

[18] Langston, A., Weiss, J., Landegger, J., Pullum, T., Morrow, M., Kabadege. M., Mugeni, C. \& Sarriot, E., Plausible role for CHW peer support groups in increasing care-seeking in an integrated community case management project in Rwanda: a mixed methods. Global Health Science and Practice, 2(3), 2014. http://dx.doi.org/10.9745/GHSP-D-14-00067

[19] Asante, A.D, Zwi, A.B., Ho, M.T., Getting by on credit: how district health managers in Ghana cope with the untimely release of funds, 2006.

[20] Aitken, J-M., Voices from the inside: managing district health services in Nepal, pp. 309-340, 1994.

[21] Lanham, H.J., Leykum Luci, K., Taylor, B.S., McCannon, C.J., Lindberg, C. \& Lester, R.T., How complexity science can inform scale-up and spread in health care: understanding the role of self-organization in variation across local contexts, pp. 194-202, 2013.

[22] Bossert, T.J., Can they get along without us? sustainability of donor-supported health projects in Central America and Africa. Social Science \& Medicine, 30(9), pp. 1015-1023, 1990. http://dx.doi.org/10.1016/0277-9536(90)90148-L 
[23] Honig, D., Navigation by Judgment: Organizational Autonomy and Country Context in the Delivery of Foreign Aid, Working Paper, Harvard University, 2014.

[24] Sarriot, E.G., Winch, P.J., Ryan, L.J., Edison, J., Bowie, J., Swedberg, E. \& Welch, R., Qualitative research to make practical sense of sustainability in primary health care projects implemented by non-governmental organizations. International Journal of Health Planning Management, 19(1), pp. 3-22, 2004.

http://dx.doi.org/10.1002/hpm.743

[25] Sarriot, E.G., Kouletio, M., Jahan, S., Rasul, I. \& Musha, A., Advancing the application of systems thinking in health: sustainability evaluation as learning and sense-making in a complex urban health system in Northern Bangladesh. Health Research Policy and Systems, 12(45), 2014.

[26] Sarriot, E. \& Kouletio, M., Community health systems as complex adaptive systems: ontology and praxis lessons from an urban health experience with demonstrated sustainability. Systemic Practice \& Action Research, pp. 1-18, 2014.

[27] Paina, L. \& Peters, D.H., Understanding pathways for scaling up health services through the lens of complex adaptive systems. Health Policy and Planning, pp. 1-9, 2011.

[28] ExpandNet, Nine steps for developing a scaling-up strategy, World Health Organization: Geneva, 2010.

[29] Simmons, R., Fajans, P. \& Ghiron, L., Scaling Up Health Service Delivery: From Pilot Innovations to Policies and Programmes, World Health Organization: Geneva, 2007.

[30] Larson, A., Ricca, J. \& Posner, J., Scaling up maternal and child health interventions in low and middle income countries: an examination of 17 case studies. presentation to the maternal and child survival project. report presented in Maternal and Child Survival Program (MCSP), 2015.

[31] Beer, S., Brain of the Firm. The Managerial Cybernetcis of Organization, John Wiley \& Sons: Chichester, UK, 1981.

[32] Jackson, D., Brady, W. \& Stein, I., Towards (re)conciliation: (re)constructing relationships between indigenous health workers and nurses. Journal Advanced Nursing, 29(1), pp. 97-103, 1999.

http://dx.doi.org/10.1046/j.1365-2648.1999.00866.x

[33] Patton, M.Q., Qualitative Evaluation and Research Methods, Sage Publications, Inc., 1990.

[34] Williams, B. \& Imam, I., Systems Concepts in Evaluation, EdgePress of Inverness: Point Reyes, 2007.

[35] Rihani, S., Complex Systems Theory and Development Practice: Understanding Non-Linear Realities, Zed Books, London, 2002.

[36] McCoy, D., Storeng, G., Filippi, V., Ronsmans, C., Osrin, D., Matthias, B., campbell, O.M., Wolfe, R., Prost, A., Hill, Z., Costello, A., Azad, K., Mwansambo, C., Manandhar, D.S., Maternal, neonataland child health interventions and services: moving from knowledge of what works to systems that deliver, pp. 87-98, 2010.

[37] Geyer, R. \& Rihani, S., Complexity and Public Policy: A New Approach to 21st Century Politics, Policy and Society, Routledge, 2010.

[38] Jackson, M., Critical systems thinking and practice. European Journal of Operational Research, 128(2), pp. 233-244, 2001. http://dx.doi.org/10.1016/S0377-2217(00)00067-9

[39] Bisits Bullen, P.A., The positive deviance/hearth approach to reducing child malnutrition: systematic review, pp. 1354-1366, 2011. 
[40] Underwood, C. \& Boulay, M., Community capacity as means to improved health practices and an end it itself: evidence from a multi-stage study, pp. 105-127, 2013.

[41] Kar, S.B, Pacual, C.A. \& Chickering, K.L., Empowerment of women for health promotion: a meta-analysis, pp. 1431-1460, 1999.

[42] Bjorkman Nyqvist, M. \& Svensson, J., Power to the people: evidence from a randomized field experiment of a community-based monitoring project in uganda, 2007.

[43] Jagosh, J., Bush, P.L., Salsberg, J., Macaulay, A.C., Greenhalgh, T., Wong, G., Cargo, M., Green, L.W., Herbert, C.P. \& Pluye, P., A realist evaluation of community-based participatory research: partnership synergy, trust building and related ripple effects, pp. 1-11, 2015.

[44] LeBan, K., How social capital in community systems strengthens health systems: people, structures, processes. In USAID; Core Group; MCHIP,Maternal and Child Health Integrated Program, pp. 1-22, 2011.

[45] Norman, C.D., Health promotion as a systems science and practice, pp. 868-872, 2009.

[46] USAID, Local Systems: a framework for supporting sustained development, Washington DC, 2014.

[47] Axelrod, R. \& Cohen, M.D., Harnessing Complexity: Organizational Implications of a Scientific Frontier, Simon and Schuster, 2001.

[48] Shiffman, J., Knowledge, moral claims and the exercise of power in global health, pp. 297299, 2014. 